\title{
SOLVABLE CROSSED PRODUCT ALGEBRAS REVISITED
}

\author{
C. BROWN AND S. PUMPLÜN
}

\begin{abstract}
For any central simple algebra over a field $F$ which contains a maximal subfield $M$ with non-trivial automorphism group $G=\operatorname{Aut}_{F}(M), G$ is solvable if and only if the algebra contains a finite chain of subalgebras which are generalized cyclic algebras over their centers (field extensions of $F$ ) satisfying certain conditions. These subalgebras are related to a normal subseries of $G$. A crossed product algebra $F$ is hence solvable if and only if it can be constructed out of such a finite chain of subalgebras. This result was stated for division crossed product algebras by Petit, and overlaps with a similar result by Albert which, however, is not explicitly stated in these terms. In particular, every solvable crossed product division algebra is a generalized cyclic algebra over $F$.
\end{abstract}

\section{INTRODUCTION}

Let $F$ be a field. A central simple algebra $A$ over $F$ of degree $n$ is a crossed product algebra if it contains a maximal subfield $M$ (i.e. with $[M: F]=n$ ) that is Galois. To be more precise, $A$ is also called a $G$-crossed product algebra, if $G=\operatorname{Gal}(M / F)$ is the Galois group of $M / F$. Crossed product algebras play an important role in the theory of central simple algebras: every element in the Brauer group of $F$ is similar to a crossed product algebra, moreover, their multiplicative structure can be described by a group action. It is well known that any central simple algebra of degree 2, 3, 4, 6 or 12 is a crossed product algebra. Moreover, any central simple algebra over a local or global field is a crossed product algebra (in that case the algebras even contain a maximal subfield that is cyclic).

Skew polynomial rings have been sucessfully used in the past to construct central simple algebras. These appear for instance as quotient algebras $D[t ; \sigma] /(f)$ when factoring out a two-sided ideal generated by a twisted polynomial $f \in D[t ; \sigma]$ with $D$ a finite-dimensional central division algebra over $F$ in [2] or [7, Sections 1.5, 1.8, 1.9]. Following Jacobson [7, p. 19], when $\left.\sigma\right|_{F}$ has finite order $m$ and $f(t)=$ $t^{m}-d \in D[t ; \sigma], d \in \operatorname{Fix}(\sigma)^{\times}$, is an invariant polynomial, such a quotient algebra is also called a generalized cyclic algebra, and denoted $(D, \sigma, d)$. In characteristic zero, generalized cyclic division algebras can be considered to be the noncommutative

2010 Mathematics Subject Classification. Primary: 16S35; Secondary: 16K20.

Key words and phrases. Skew polynomial ring, skew polynomial, solvable crossed product algebra, generalized cyclic algebra, cyclic subalgebra, crossed product subalgebra, admissible group. 
analogue of simple algebraic field extensions. To our knowledge, generalized cyclic division algebras appear for the first time in a paper by Amitsur [2], where they are indeed called noncommutative cyclic fields. They are examples of crossed products of central simple algebras which were introduced by Teichmüller [17] in 1940.

In this paper, we will revisit a result on the structure of crossed product algebras with solvable Galois group due to both Albert [1, p. 182-187] and Petit [11, Section 7].

To be more precise, we write up the proof for Albert's result following the approach given by Petit, i.e. using generalized cyclic algebras (none of Petit's results are proved). In the process, we generalize some results to central simple algebras which need neither be crossed products nor division algebras. In order to do so, we extend the classical definition of a generalized cyclic algebra $(D, \sigma, d)$ as we do not assume that $D$ needs to be a division algebra.

As a special case we obtain that a $G$-crossed product algebra is solvable if and only if it can be constructed as a finite chain of subalgebras over $F$ which are generalized cyclic algebras over their centers, which are field extensions of $F$. The generalized cyclic algebras appearing in this chain correspond to the normal subgroups in a chain of normal subgroups of the solvable group $G$. We highlight how the structure of the solvable group (i.e., its chain of normal subgroups $G_{i}$ ) is connected to the structure of the algebra, and how each subalgebra is related to a normal subgroup $G_{i}$ in the chain and the order of the factor groups $G_{i+1} / G_{i}$.

The paper is structured as follows. After the basic terminology in Section 1 we look at the existence of crossed product algebras and in particular, of cyclic algebras, inside central simple algebras in Section 2. As a byproduct, we show that even if a central division algebra $A$ over $F$ is a noncrossed product, if it contains a maximal field extension $M$ with a non-trivial $\sigma \in G=\operatorname{Aut}_{F}(M)$ of order $h$, then it contains a cyclic division algebra of degree $h$, and a crossed product algebra $(M, G, \mathfrak{a})$ of degree $|G|$ as well, both of them not necessarily with center $F$, however (Theorem 4).

The first results on the structure of central simple algebras which contain a maximal subfield with non-trivial solvable group $G=\operatorname{Aut}_{F}(M)$ are stated in Section [3] (Theorems 7 and 13). These algebras have certain chains of generalized cyclic algebras (with centers larger than $F$ ) as subalgebras.

As a consequence, we can show in Section 4 that all solvable crossed product algebras can be constructed as chains of such generalized cyclic algebras and that if a central simple algebra contains a maximal subfield with $G=\operatorname{Aut}_{F}(M)$ that this $G$ is solvable exactly if there is such a chain (Theorems 14 and Corollary 16). In particular, every solvable $G$-crossed product division algebra is a generalized cyclic algebra (Corollary 18). Some straightforward applications to admissible groups are given in Section [5. In Section 6 we generalize a result on crossed product algebras 
with Galois group $G \cong \mathbb{Z}_{2} \times \mathbb{Z}_{2}$ by Albert [1, p. 186], cf. also [7, Theorem 2.9.55], to crossed product algebras with $G$ any abelian group, and give a recipe how to construct central division algebras containing a given Galois field extension with abelian Galois group from a chain of generalized cyclic algebras, complementing the construction of such algebras via generic algebras by Amitsur and Saltman described in [7, 4.6].

Most of the results presented here are part of the first author's PhD thesis [4] written under the supervision of the second author.

\section{Preliminaries}

1.1. Twisted polynomial rings and (nonassociative) algebras. In the following, we recall some results from [7] and [11] for the convenience of the reader.

Let $S$ be a unital (associative, not necessarily commutative) ring and $\sigma$ an injective ring endomorphism of $S$. The twisted polynomial ring $R=S[t ; \sigma]$ is the set of twisted polynomials

$$
a_{0}+a_{1} t+\cdots+a_{n} t^{n}
$$

with $a_{i} \in S$, where addition is defined term-wise and multiplication by

$$
t a=\sigma(a) t \quad(a \in S) .
$$

For $f=a_{0}+a_{1} t+\cdots+a_{n} t^{n}$ with $a_{n} \neq 0$ define $\operatorname{deg}(f)=n$ and $\operatorname{deg}(0)=-\infty$. An element $f \in R$ is irreducible in $R$ if it is not a unit and it has no proper factors, i.e if there do not exist $g, h \in R$ with $\operatorname{deg}(g), \operatorname{deg}(h)<\operatorname{deg}(f)$ such that $f=g h$. An element $f \in R$ is called invariant (or two-sided) if $R f$ is a two-sided ideal in $R$.

We now briefly explain how classical quotient algebras $R / R f, f$ invariant, fit into the nonassociative setting of Petit's paper [11]:

In the following, we always assume that $f(t) \in S[t ; \sigma]$ is monic of degree $m>1$. Then for all $g, f \in R, g \neq 0$, there exist unique $r, q \in R$ such that $\operatorname{deg}(r)<\operatorname{deg}(f)$ and

$$
g=q f+r
$$

e.g. see [13].

In [11] and [13, it is shown that the additive group $S_{f}=\{g \in R \mid \operatorname{deg}(g)<m\}$ of twisted polynomials of degree less that $m$ is a nonassociative unital ring together with the multiplication given by

$$
g \circ h=g h \bmod _{r} f
$$

where $\bmod _{r} f$ denotes the remainder $\bmod _{r} f$ of right division by $f$. This algebra is also denoted by $R / R f$.

Note that since the remainders are uniquely determined, the elements in the set $S_{f}$ also canonically represent the elements of the left $S[t ; \sigma]$-module $S[t ; \sigma] / S[t ; \sigma] f$. 
$S_{0}=\left\{a \in S \mid a h=h a\right.$ for all $\left.h \in S_{f}\right\}$ is a commutative subring of $S$, and $S_{f}$ is a unital algebra over $S_{0}$. If $S$ is a division ring, the structure of $S_{f}$ is extensively investigated in [11], else see [13]. For instance, if $S$ is a division ring and the $S_{0^{-}}$ algebra $S_{f}$ is finite-dimensional, then $S_{f}$ is a division algebra if and only if $f(t)$ is irreducible [11, (9)]. In the following, we will only be interested in the case that $S_{f}$ is a unital associative algebra, which happens if and only if $f$ is an invariant polynomial in $R$, i.e. generates a two-sided ideal $R f$ in $R$ [11]. In that case, $S_{f}=R / R f$ is the well known quotient algebra obtained by factoring out the two-sided ideal in $R$ generated by $f$.

We will moreover only need the case that $S$ is a finite-dimensional algebra over a field $F$ with center $F$ and only consider automorphisms $\sigma$ of $S$ such that $\left.\sigma\right|_{F}$ has finite order $m$. Then, by the Theorem of Skolem-Noether, $\sigma^{m}$ is an inner automorphism $I_{u}(y)=u y u^{-1}$ of $S$ [7, Sec. 1.4].

1.2. Generalized cyclic algebras and generalized cyclic extensions. Let $S$ be a finite-dimensional simple algebra of degree $n$ over its center $F=C(S)$, and $\sigma \in \operatorname{Aut}(S)$ such that $\left.\sigma\right|_{F}$ has finite order $m$ and fixed field $F_{0}=\operatorname{Fix}(\sigma)$.

Generalizing Jacobson's definition [7, p. 19], which assumes that $S$ is a division algebra, we define a generalized cyclic algebra as an associative algebra of the type $S_{f}=S[t ; \sigma] / S[t ; \sigma] f(t)$ which is constructed using an invariant twisted polynomial

$$
f(t)=t^{m}-d \in S[t ; \sigma]
$$

with $d \in \operatorname{Fix}(\sigma)^{\times}$non-zero.

We write $S_{f}=(S, \sigma, d)$ for this algebra. $(S, \sigma, d)$ is a central simple algebra over $F_{0}=\operatorname{Fix}(\sigma)$ of degree $m n$ and the centralizer of $S$ in $(S, \sigma, d)$ is $F$ ([7, p. 20] if $S$ is division, else [18]).

Note that this definition canonically generalizes the one of a cyclic algebra $\left(F / F_{0}, \sigma, d\right)$, where $f(t)=t^{m}-d \in F[t ; \sigma]$, and $F / F_{0}$ is a cyclic Galois extension of degree $m$ with Galois group $G=\langle\sigma\rangle$. This is the algebra $S_{f}=F[t ; \sigma] / F[t ; \sigma]\left(t^{m}-d\right)$, cf. [7, p. 19] or [11, p. 13-13]. This case appears when $S=F$ above.

Generalized cyclic algebras are a special case of generalized crossed products, i.e. crossed products of simple algebras cf. for instance [5, p. 35], [8], [18]. We will mostly need crossed products involving Galois fields:

1.3. Crossed product algebras. Let $F$ be a field and $A$ be a (finite-dimensional) central simple algebra over $F$ of degree $n$. $A$ is called a $G$-crossed product algebra or crossed product algebra if it contains a maximal field extension $K / F$ which is Galois with Galois group $G=\operatorname{Gal}(K / F)$.

Equivalently, we can define a $(G$-)crossed product algebra $(M, G, \mathfrak{a})$ over $F$ via factor sets starting with a finite Galois field extension as follows: Take a finite Galois 
field extension $M / F$ of degree $n$ with Galois group $G$. Suppose $\left\{a_{\sigma, \tau} \mid \sigma, \tau \in G\right\}$ is a set of elements of $M^{\times}$such that

$$
a_{\sigma, \tau} a_{\sigma \tau, \rho}=a_{\sigma, \tau \rho} \sigma\left(a_{\tau, \rho}\right),
$$

for all $\sigma, \tau, \rho \in G$. Then a map $\mathfrak{a}: G \times G \rightarrow M^{\times},(\sigma, \tau) \mapsto a_{\sigma, \tau}$, is called a factor set or 2-cocycle of $G$.

An associative multiplication is defined on the $F$-vector space $\bigoplus_{\sigma \in G} M x_{\sigma}$ by

$$
\begin{aligned}
& x_{\sigma} m=\sigma(m) x_{\sigma}, \\
& x_{\sigma} x_{\tau}=a_{\sigma, \tau} x_{\sigma \tau},
\end{aligned}
$$

for all $m \in M, \sigma, \tau \in G$. This way $\bigoplus_{\sigma \in G} M x_{\sigma}$ becomes an associative central simple $F$-algebra that contains a maximal subfield isomorphic to $M$. This algebra is denoted by $(M, G, \mathfrak{a})$ and is a $G$-crossed product algebra over $F$. If $G$ is solvable then $A$ is also called a solvable $G$-crossed product.

In the following, we will only consider unital algebras $A$ over a field $F$ which are finite-dimensional without explicitly saying so. We denote the set of invertible elements of $A$ by $A^{\times}$.

\section{Cyclic and Crossed product subalgebras of Central simple}

\section{ALGEBRAS}

In this section, let $M / F$ be a field extension of degree $n$, and $G=\operatorname{Aut}_{F}(M)$ the group of automorphisms of $M$ which fix the elements of $F$. Let $A$ be a central simple algebra of degree $n$ over $F$ and suppose that $M$ is contained in $A$, i.e. is a maximal subfield of $A$.

The results in this section are stated for central division algebras $A$ over $F$ for instance in [11, and none of them are proved there. We generalize them to any central simple algebra $A$ with a maximal subfield $M$ as above, so that [11, (26)] which is well known for Galois extensions $M / F$ becomes:

Lemma 1. (i) For any $\sigma \in G$ there exists an invertible $x_{\sigma} \in A$ such that the inner automorphism

$$
I_{x_{\sigma}}: A \rightarrow A, y \mapsto x_{\sigma} y x_{\sigma}^{-1}
$$

restricted to $M$ is $\sigma$.

(ii) Given any $\sigma \in G$, we have

$$
\left\{x \in A^{\times}\left|I_{x}\right|_{M}=\sigma\right\}=M^{\times} x_{\sigma} .
$$

(iii) The set of cosets $\left\{M^{\times} x_{\sigma} \mid \sigma \in G\right\}$ together with the multiplication given by

$$
M^{\times} x_{\sigma} M^{\times} x_{\tau}=M^{\times} x_{\sigma \tau}
$$

is a group isomorphic to $G$, where $\sigma$ and $M^{\times} x_{\sigma}$ correspond under this isomorphism. 
Proof. (i) By the Theorem of Skolem-Noether, there exists $x_{\sigma} \in A^{\times}$such that $\left.I_{x_{\sigma}}\right|_{M}=\sigma$.

(ii) We have

$$
I_{\left(m x_{\sigma}\right)}(y)=\left(m x_{\sigma}\right) y\left(m x_{\sigma}\right)^{-1}=\left(m x_{\sigma}\right) y\left(x_{\sigma}^{-1} m^{-1}\right)=m \sigma(y) m^{-1}=\sigma(y),
$$

for all $m, y \in M^{\times}$, and thus $M^{\times} x_{\sigma} \subset\left\{x \in A^{\times}\left|I_{x}\right|_{M}=\sigma\right\}$.

Suppose $u \in\left\{x \in A^{\times}\left|I_{x}\right|_{M}=\sigma\right\}$. As $u$ and $x_{\sigma}$ are invertible, we can write $u=v x_{\sigma}$ for some $v \in A^{\times}$. We still have to prove that $v \in M^{\times}$. We have

$$
\sigma(y)=I_{u}(y)=\left(v x_{\sigma}\right) y\left(v x_{\sigma}\right)^{-1}=v x_{\sigma} y x_{\sigma}^{-1} v^{-1}=v \sigma(y) v^{-1},
$$

for all $y \in M$, and so $\sigma(y) v=v \sigma(y)$ for all $y \in M$, that is $m v=v m$ for all $m \in M$ since $\sigma$ is bijective. Therefore $v$ is contained in the centralizer of $M$ in $A$, which is equal to $M$ because $M$ is a maximal subfield of $A$.

(iii) Let $s=m_{1} x_{\sigma} m_{2} x_{\tau} \in M^{\times} x_{\sigma} M^{\times} x_{\tau}$ for some $m_{1}, m_{2} \in M^{\times}, \sigma, \tau \in G$. Then

$$
\begin{aligned}
I_{s}(y) & =\left(m_{1} x_{\sigma} m_{2} x_{\tau}\right) y\left(m_{1} x_{\sigma} m_{2} x_{\tau}\right)^{-1} \\
& =m_{1} x_{\sigma} m_{2}\left(x_{\tau} y x_{\tau}^{-1}\right) m_{2}^{-1} x_{\sigma}^{-1} m_{1}^{-1} \\
& =m_{1} x_{\sigma}\left(m_{2} \tau(y) m_{2}^{-1}\right) x_{\sigma}^{-1} m_{1}^{-1} \\
& =m_{1}\left(x_{\sigma} \tau(y) x_{\sigma}^{-1}\right) m_{1}^{-1} \\
& =m_{1} \sigma(\tau(y)) m_{1}^{-1} \\
& =\sigma(\tau(y))=\sigma \tau(y),
\end{aligned}
$$

for all $y \in M$, which means $I_{s}$ restricts to $\sigma \tau$ on $M$. Therefore $m_{1} x_{\sigma} m_{2} x_{\tau} \in M^{\times} x_{\sigma \tau}$ by (ii) and so $M^{\times} x_{\sigma} M^{\times} x_{\tau} \subseteq M^{\times} x_{\sigma \tau}$. In particular, we get $x_{\sigma} x_{\tau}=m x_{\sigma \tau}$ for some $m \in M^{\times}$. Thus

$$
l x_{\sigma \tau}=l m^{-1} x_{\sigma} x_{\tau} \in M^{\times} x_{\sigma} M^{\times} x_{\tau}
$$

for all $l \in M^{\times}$, i.e. $M^{\times} x_{\sigma \tau} \subseteq M^{\times} x_{\sigma} M^{\times} x_{\tau}$, and hence $M^{\times} x_{\sigma \tau}=M^{\times} x_{\sigma} M^{\times} x_{\tau}$.

Finally, the map $\left\{M^{\times} x_{\sigma} \mid \sigma \in G\right\} \rightarrow G, M^{\times} x_{\sigma} \mapsto \sigma$, is clearly bijective and is multiplicative by (4) which yields the assertion.

The following generalizes [11, (27)] to central simple algebras with a maximal subfield $M$ as above. The result was again only stated for division algebras and also not in terms of crossed product algebras:

Theorem 2. (i) A contains a subalgebra $M(G)$ which is a crossed product algebra $(M, G, \mathfrak{a})$ of degree $|G|$ over $\operatorname{Fix}(G)$ with maximal subfield $M$.

(ii) $A=M(G)$ if and only if $M$ is a Galois field extension of $F$. In that case, $A$ is a $G$-crossed product algebra over $F$.

(iii) For any subgroup $H$ of $G$, there is a subalgebra $M(H)$ of both $M(G)$ and $A$ which is a $H$-crossed product algebra of degree $|H|$ over $\operatorname{Fix}(H)$ with maximal subfield M. 
Proof. (i) There is an $F$-subalgebra $M(G)$ of $A$ admitting a basis $\left\{x_{\sigma} \mid \sigma \in G\right\}$ as a vector space over $M$ : Let $M(G)$ denote the subset of $A$ which is generated as an $M$-vector space by $\left\{x_{\sigma} \mid \sigma \in G\right\}$. Note that $1 \in M^{\times} x_{i d} \subset M(G)$ and also $x_{\sigma} x_{\tau} \in M^{\times} x_{\sigma \tau}$ for all $\sigma, \tau \in G$ by Lemma 1, In particular, there exist $a_{\sigma, \tau} \in M^{\times}$ such that

$$
x_{\sigma} x_{\tau}=a_{\sigma, \tau} x_{\sigma \tau}
$$

holds for all $\sigma, \tau \in G$. Therefore $M(G)$ is closed under multiplication, contains the identity, and can easily be seen to be an $F$-subalgebra of $A$.

Furthermore, $\sigma(m)=I_{x_{\sigma}}(m)=x_{\sigma} m x_{\sigma}^{-1}$ for all $m \in M$ by Lemma 1 which yields

$$
x_{\sigma} m=\sigma(m) x_{\sigma},
$$

for all $m \in M, \sigma, \tau \in G$.

The set $\left\{x_{\sigma} \mid \sigma \in G\right\}$ is linearly independent over $M$ : Suppose

$$
\sum_{\sigma \in G} m_{\sigma} x_{\sigma}=0
$$

for some $m_{\sigma} \in M$, not all 0 , where the sum (7) is chosen so that the number of non-zero $m_{\sigma}$ is minimal. Let $\tau \in G$ be such that $m_{\tau} \neq 0$, then

$$
0=\left(\sum_{\sigma \in G} m_{\sigma} x_{\sigma}\right) m-\tau(m)\left(\sum_{\sigma \in G} m_{\sigma} x_{\sigma}\right)=\sum_{\sigma \in G} m_{\sigma}(\sigma(m)-\tau(m)) x_{\sigma},
$$

for all $m \in M$ by (6) and (7). The coefficient of $x_{\tau}$ in (8) is 0 , so by the minimality of (7) we obtain

$$
m_{\sigma}(\sigma(m)-\tau(m))=0
$$

for all $\sigma \in G$. This means $\sigma=\tau$ for all $\sigma \in G$ with $m_{\sigma} \neq 0$, a contradiction, so we proved linear independency.

$M / \operatorname{Fix}(G)$ is a Galois field extension of degree $|G|$, and the associativity of $M(G)$ implies in particular $\left(x_{\sigma} x_{\tau}\right) x_{\rho}=x_{\sigma}\left(x_{\tau} x_{\rho}\right)$ for all $\sigma, \tau, \rho \in G$. This means

$$
a_{\sigma, \tau} a_{\sigma \tau, \rho}=a_{\sigma, \tau \rho} \sigma\left(a_{\tau, \rho}\right),
$$

for all $\sigma, \tau, \rho \in G$. Therefore the constants $a_{\sigma, \tau}$ define a factor set

$$
\mathfrak{a}: G \times G \rightarrow M^{\times},(\sigma, \tau) \mapsto a_{\sigma, \tau}
$$

of $G$ and hence $M(G)$ is the $G$-crossed product algebra $(M, G, \mathfrak{a})$ over $\operatorname{Fix}(G)$ of degree $|G|$.

(ii) We have $[M: F]=n$ and $A$ has dimension $n^{2}$ over $F$. If $M$ is not a Galois extension of $F$, then $|G|<n$ and thus $\left\{x_{\sigma} \mid \sigma \in G\right\}$ cannot be a set of generators for $A$ as a vector space over $M$. Conversely, if $M / F$ is a Galois extension, then $|G|=n$ and since $\left\{x_{\sigma} \mid \sigma \in G\right\}$ is linearly independent over $M$, counting dimensions yields $M(G)=A$. The rest of the assertion is trivial.

(iii) For any subgroup $H$ of $G$, there is an $F$-subalgebra $M(H)$ of $M(G)$ with basis 
$\left\{x_{\sigma} \mid \sigma \in H\right\}$ as a vector space over $M$ and multiplication in $M(H)$ defined by constants $a_{\sigma, \tau} \in M^{\times}$for all $m \in M, \sigma, \tau \in H$ according to the rules in (i): clearly $M(H)$ is closed under multiplication since if $x_{\sigma}, x_{\tau} \in M(H)$, then $\sigma, \tau \in H$, hence also $\sigma \tau \in H$ and so $x_{\sigma \tau} \in M(H)$. Additionally $1 \in M^{\times} x_{i d} \subset M(H)$, and thus $M(H)$ is a subalgebra of $M(G) . M / \operatorname{Fix}(H)$ is a Galois field extension of degree $|H|$ and with the same argument as in the proof of (i) thus $M(H)$ is a $H$-crossed product algebra over $\operatorname{Fix}(H)$ of degree $|H|$.

More precisely, a closer look at the above proof reveals:

Lemma 3. (i) For any subgroup $H$ of $G, M(H)$ is a $H$-crossed product algebra over its center with

$$
M(H)=\left(M, H, \mathfrak{a}_{H}\right),
$$

where $\mathfrak{a}_{H}$ denotes the factor set $\mathfrak{a}$ of the crossed product algebra $M(G)=(M, G, \mathfrak{a})$, restricted to the elements in $H$.

(ii) If $H$ is a cyclic subgroup of $G$ of order $h>1$ generated by $\sigma \in G$, then there exists $c \in \operatorname{Fix}(\sigma)^{\times}$such that

$$
M(H) \cong M[t ; \sigma] / M[t ; \sigma]\left(t^{h}-c\right)
$$

is a cyclic algebra of degree $h$ over $\operatorname{Fix}(\sigma)$ and an F-subalgebra of $A$.

Proof. (i) is trivial.

(ii) $M(H)$ is a $H$-crossed product algebra $\left(M, H, \mathfrak{a}_{H}\right)$ over $\operatorname{Fix}(\sigma)$ of degree $h$ by Theorem 2 and $H$ is a cyclic group, therefore $M(H)$ is a cyclic algebra over $\operatorname{Fix}(\sigma)$ of degree $h$, i.e. there exists $c \in \operatorname{Fix}(\sigma)^{\times}$such that $M(H) \cong M[t ; \sigma] / M[t ; \sigma]\left(t^{h}-c\right)$, e.g. see [7, p. 19].

We conclude that even if a central division algebra $A$ over $F$ is a noncrossed product, if $A$ contains a maximal field extension $M$ with a non-trivial $\sigma \in G=$ $\operatorname{Aut}_{F}(M)$ of order $h$, then it contains a cyclic division algebra of degree $h$ (though generally not with center $F$ ):

Theorem 4. Let $A$ be a central division algebra over $F$ with maximal subfield $M$ and non-trivial $\sigma \in G=\operatorname{Aut}_{F}(M)$ of order $h$. Then $A$ contains the cyclic division algebra

$$
(M / \operatorname{Fix}(\sigma), \sigma, c)=M[t ; \sigma] / M[t ; \sigma]\left(t^{h}-c\right)
$$

of degree $h$ over $\operatorname{Fix}(\sigma)$ as an F-subalgebra.

This generalizes [11, (28)] to central simple algebras with a maximal subfield $M$ such that $G=\operatorname{Aut}_{F}(M)$ is not trivial. 
Remark 5. The question when a central division algebra $A$ over $F$ has a cyclic subalgebra of prime degree was recently raised in [10, Question 1]. If $F$ is a Henselian field such that $\bar{F}$ is a global field, and $A$ is an central division algebra over $F$ such that $\operatorname{char}(\bar{F})$ does not divide $\operatorname{deg}(A)$, then $A$ contains a cyclic division algebra of prime degree [10, Theorem 3].

By Theorem 4, any central division algebra $A$ over $F$ containing a maximal subfield $M$ with some $\sigma \in G=\operatorname{Aut}_{F}(M)$ of prime order $p$ contains a cyclic division algebra of prime degree $p$.

A central division algebra of prime degree over $F$ is cyclic if and only if it has a cyclic subalgebra of prime degree (not necessarily with center $F$ ) [10, p. 2]. Theorem 4 yields the following observations:

Corollary 6. Let $A$ be a central division algebra over $F$.

(i) If $A$ has prime degree then either $A$ is a cyclic algebra or each of its maximal subfields $M$ has trivial automorphism group $\operatorname{Aut}_{F}(M)$.

(ii) Suppose $A$ contains a maximal subfield $M$ such that $G=\operatorname{Aut}_{F}(M)$ is nontrivial. Then $A$ contains the $G$-crossed product division algebra $M(G)=(M, G, \mathfrak{a})$ of degree $|G|$ over $\operatorname{Fix}(G)$ as a subalgebra.

Proof. (i) If $G=\operatorname{Aut}_{F}(M)$ is non-trivial, then $A$ contains the cyclic algebra $M(G)$ of degree $|G| \leq p$ over $F_{0}=\operatorname{Fix}(G)$ as subalgebra. Since $M / F_{0}$ is a maximal subfield of $M(G)$, it also has degree $|G|$. Looking at the possible degrees of the intermediate field extensions of $M / F$ we have $\left[M: F_{0}\right]=1$ or $\left[M: F_{0}\right]=p$, so $|G|=1$ or $|G|=p$. If $|G|=p$ then $A$ is a cyclic algebra. Hence if $A$ is not cyclic then each of its maximal subfields $M$ must have trivial automorphism group $\operatorname{Aut}_{F}(M)$.

(ii) is trivial.

\section{Central simple algebras containing maximal subfields with a SOLVABLE $F$-AUTOMORPHISM GROUP}

Let $G$ be finite a solvable group, i.e. there exists a chain of subgroups

$$
\{1\}=G_{0} \leq G_{1} \leq \ldots \leq G_{k}=G,
$$

such that $G_{j}$ is normal in $G_{j+1}$ and $G_{j+1} / G_{j}$ is cyclic of prime order $q_{j}$ for all $j \in\{0, \ldots, k-1\}$, that is

$$
G_{j+1} / G_{j}=\left\{G_{j}, G_{j} \sigma_{j+1}, \ldots\right\},
$$

for some $\sigma_{j+1} \in G_{j+1}$. Lemma 1. Theorem 2 and Corollary 3 yield the following generalization of [11, (29)], which only claims the result for central division algebras over $F$ : 
Theorem 7. Let $M / F$ be a field extension of degree $n$ with non-trivial solvable $G=\operatorname{Aut}_{F}(M)$, and $A$ a central simple algebra of degree $n$ over $F$ with maximal subfield $M$. Then there exists a chain of subalgebras

$$
M=A_{0} \subset A_{1} \subset \ldots \subset A_{k}=M(G) \subset A,
$$

of $A$ which are $G_{i}$-crossed product algebras over $Z_{i}=\operatorname{Fix}\left(G_{i}\right)$ and where

$$
A_{i+1} \cong A_{i}\left[t_{i} ; \tau_{i}\right] / A_{i}\left[t_{i} ; \tau_{i}\right]\left(t_{i}^{q_{i}}-c_{i}\right)
$$

for all $i \in\{0, \ldots, k-1\}$, such that

(i) $q_{i}$ is the prime order of the factor group $G_{i+1} / G_{i}$ in the chain of normal subgroups (9),

(ii) $\tau_{i}$ is an $F$-automorphism of $A_{i}$ of inner order $q_{i}$ which restricts to the automorphism $\sigma_{i+1} \in G_{i+1}$ that generates $G_{i+1} / G_{i}$, and

(iii) $c_{i} \in \operatorname{Fix}\left(\tau_{i}\right)$ is invertible.

Note that the inclusion $M(G) \subset A$ in (11) is an equality if and only if $M / F$ is a Galois extension by Theorem 2, i.e. if and only if $A$ is a $G$-crossed product algebra.

Proof. Define $A_{i}=M\left(G_{i}\right)$ for all $i \in\{1, \ldots, k\} . A_{i}$ is a $G_{i}$-crossed product algebra over $\operatorname{Fix}\left(G_{i}\right)$ by Theorem 2 ,

$G_{1} / G_{0} \cong G_{1}$ is a cyclic subgroup of $G$ of order $q_{0}$ generated by some $\sigma_{1} \in G$. Let $\tau_{0}=\sigma_{1}$, then there exists $c_{0} \in \operatorname{Fix}\left(\tau_{0}\right)$ such that $A_{1}=M\left(G_{1}\right)$ is $F$-isomorphic to

$$
M\left[t_{0} ; \tau_{0}\right] / M\left[t_{0} ; \tau_{0}\right]\left(t_{0}^{q_{0}}-c_{0}\right),
$$

by Corollary 3, which is a cyclic algebra of prime degree $q_{0}$ over $\operatorname{Fix}\left(\tau_{0}\right)$.

Now $G_{1} \triangleleft G_{2}$ and $G_{2} / G_{1}$ is cyclic of prime order $q_{1}$ with

$$
G_{2} / G_{1}=\left\{G_{1}, G_{1} \sigma_{2}, \ldots, G_{1} \sigma_{2}^{q_{1}-1}\right\}
$$

for some $\sigma_{2} \in G_{2}$. Hence we can write $G_{2}=\left\{h \sigma_{2}^{i} \mid h \in G_{1}, 0 \leq i \leq q_{1}-1\right\}$ and thus $A_{2}=M\left(G_{2}\right)$ has a basis

$$
\left\{x_{h \sigma_{2}^{j}} \mid h \in G_{1}, 0 \leq j \leq q_{1}-1\right\},
$$

as an $M$-vector space. Recall

$$
M^{\times} x_{h \sigma_{2}^{j}}=M^{\times} x_{h} x_{\sigma_{2}^{j}}=M^{\times} x_{h} x_{\sigma_{2}}^{j}
$$

for all $h \in G_{1}$ by Lemma 1, and $\left\{1, x_{\sigma_{2}}, \ldots, x_{\sigma_{2}}^{q_{1}-1}\right\}$ is a basis for $A_{2}$ as a left $A_{1^{-}}$ module, i.e.

$$
A_{2}=A_{1}+A_{1} x_{\sigma_{2}}+\ldots+A_{1} x_{\sigma_{2}}^{q_{1}-1} .
$$


We have $G_{2} G_{1}=G_{1} G_{2}$ as $G_{1}$ is normal in $G_{2}$ and so for every $h \in G_{1}$, we get $\sigma_{2} h=h^{\prime} \sigma_{2}$ for some $h^{\prime} \in G_{1}$. Choose the basis $\left\{x_{h} \mid h \in G_{1}\right\}$ of $A_{1}$ as a vector space over $M$. By (5) we obtain

$$
x_{\sigma_{2}} x_{h}=a_{\sigma_{2}, h} x_{\sigma_{2} h}=a_{\sigma_{2}, h} x_{h^{\prime} \sigma_{2}}=a_{\sigma_{2}, h}\left(a_{h^{\prime}, \sigma_{2}}\right)^{-1} x_{h^{\prime}} x_{\sigma_{2}} .
$$

Recall $x_{\sigma_{2}} \in A^{\times}$by Lemma 1, The inner automorphism

$$
\tau_{1}: A \rightarrow A, z \mapsto x_{\sigma_{2}} z x_{\sigma_{2}}^{-1}
$$

restricts to $\sigma_{2}$ on $M$. Moreover,

$$
\begin{aligned}
\tau_{1}\left(x_{h_{j}}\right) & =x_{\sigma_{2}} x_{h_{j}} x_{\sigma_{2}}^{-1}=a_{\sigma_{2}, h_{j}}\left(a_{h_{j}^{\prime}, \sigma_{2}}\right)^{-1} x_{h_{j}^{\prime}} x_{\sigma_{2}} x_{\sigma_{2}}^{-1} \\
& =a_{\sigma_{2}, h_{j}}\left(a_{h_{j}^{\prime}, \sigma_{2}}\right)^{-1} x_{h_{j}^{\prime}} \in A_{1},
\end{aligned}
$$

for all $h_{j} \in G_{1}$, i.e. $\left.\tau_{1}\right|_{A_{1}}(y) \in A_{1}$ for all $y \in A_{1}$ and so $\left.\tau_{1}\right|_{A_{1}}$ is an $F$-automorphism of $A_{1}$. Moreover,

$$
x_{\sigma_{2}} x_{h}=\left.\tau_{1}\right|_{A_{1}}\left(x_{h}\right) x_{\sigma_{2}},
$$

for all $h \in G_{1}$ by (15), (16), and

$$
x_{\sigma_{2}} m=\sigma_{2}(m) x_{\sigma_{2}}=\left.\tau_{1}\right|_{A_{1}}(m) x_{\sigma_{2}},
$$

for all $m \in M$. We conclude that

$$
x_{\sigma_{2}} y=\left.\tau_{1}\right|_{A_{1}}(y) x_{\sigma_{2}}
$$

for all $y \in A_{1}$. Define $c_{1}=x_{\sigma_{2}}^{q_{1}}$, then $\sigma_{2}^{q_{1}} \in G_{1}$ by (10) which implies $c_{1} \in A_{1}$. Furthermore $c_{1}$ is invertible since $x_{\sigma_{2}}$ is invertible. Also,

$$
\left.\tau_{1}\right|_{A_{1}}\left(c_{1}\right)=x_{\sigma_{2}} x_{\sigma_{2}}^{q_{1}} x_{\sigma_{2}}^{-1}=c_{1}
$$

which means $c_{1} \in \operatorname{Fix}\left(\left.\tau_{1}\right|_{A_{1}}\right)^{\times}$. Notice

$$
x_{\sigma_{2}^{-q_{1}}} x_{\sigma_{2}^{q_{1}}}=a_{\sigma_{2}^{-q_{1}}, \sigma_{2}^{q_{1}}} x_{\mathrm{id}} \in M^{\times},
$$

therefore $c_{1}^{-1}=x_{\sigma_{2}^{q_{1}}}^{-1} \in M^{\times} x_{\sigma_{2}^{-q_{1}}} \in A_{1}$ as $\sigma_{2}^{-q_{1}} \in G_{1}$. Hence $\left.\tau_{1}\right|_{A_{1}}$ has inner order $q_{1}$, since indeed

$$
\left(\left.\tau_{1}\right|_{A_{1}}\right)^{q_{1}}: A_{1} \rightarrow A_{1}, z \mapsto c_{1} z c_{1}^{-1},
$$

is an inner automorphism.

Consider the algebra

$$
B_{2}=A_{1}\left[t_{1} ;\left.\tau_{1}\right|_{A_{1}}\right] / A_{1}\left[t_{1} ;\left.\tau_{1}\right|_{A_{1}}\right]\left(t_{1}^{q_{1}}-c_{1}\right)
$$

with center

$$
C\left(B_{2}\right) \supset\left\{b \in A_{1} \mid b h=h b \text { for all } h \in B_{2}\right\}=C\left(A_{1}\right) \cap \operatorname{Fix}\left(\tau_{1}\right) \supset F .
$$

By (14) and (17), the $F$-linear map

$$
\phi: A_{2} \rightarrow B_{2}, y x_{\sigma_{2}}^{i} \mapsto y t_{1}^{i} \quad\left(y \in A_{1}\right),
$$


is an isomorphism. In addition, by a straightforward calculation we have

$$
\phi\left(\left(y x_{\sigma_{2}}^{i}\right)\left(z x_{\sigma_{2}}^{j}\right)\right)=\phi\left(y x_{\sigma_{2}}^{i}\right) \phi\left(z x_{\sigma_{2}}^{j}\right)
$$

for all $y, z \in A_{1}, i, j \in\left\{0, \ldots, q_{1}-1\right\}$, so $\phi$ is also multiplicative, thus an $F$-algebra isomorphism. Continuing in this manner for $G_{2} \triangleleft G_{3}$ etc. yields the assertion.

For a subset $B$ in $A$, let $\operatorname{Cent}_{A}(B)$ denote the centralizer of $B$ in $A$. Then the algebras $A_{i}$ are the centralizers of $\operatorname{Fix}\left(G_{i}\right)$ in $A_{i+1}$ :

Corollary 8. Let $M / F$ be a field extension of degree $n$ with non-trivial solvable $G=\operatorname{Aut}_{F}(M)$ with normal series (9), and $A$ a central simple algebra of degree $n$ over $F$ with maximal subfield $M$. Then

$$
A_{i}=\operatorname{Cent}_{A_{i+1}}\left(\operatorname{Fix}\left(G_{i}\right)\right)
$$

for all $i \in\{0, \ldots, k-1\}$ where $A_{i}=M\left(G_{i}\right)$ are as in Theorem 7 .

Proof. Clearly $A_{i} \subset \operatorname{Cent}_{A_{i+1}}\left(\operatorname{Fix}\left(G_{i}\right)\right)$ for all $i \in\{0, \ldots, k-1\}$ because $A_{i} \subset A_{i+1}$ and $C\left(A_{i}\right)=\operatorname{Fix}\left(G_{i}\right)$. To prove $\operatorname{Cent}_{A_{i+1}}\left(\operatorname{Fix}\left(G_{i}\right)\right) \subset A_{i}$, let $x=\sum_{\sigma \in G_{i+1}} m_{\sigma} x_{\sigma} \in$ $\operatorname{Cent}_{A_{i+1}}\left(\operatorname{Fix}\left(G_{i}\right)\right)$ for some $m_{\sigma} \in M$. If $\tau \in G_{i+1} \backslash G_{i}$ is such that $m_{\tau} \neq 0$, then $m_{\tau} x_{\tau} z=z m_{\tau} x_{\tau}$ for all $z \in \operatorname{Fix}\left(G_{i}\right)$, that is, $\tau(z)=z$ for all $z \in \operatorname{Fix}\left(G_{i}\right)$.

Now $\operatorname{Fix}\left(G_{i+1}\right)$ is properly contained in $\operatorname{Fix}\left(G_{i}\right)$, therefore $\tau(z) \neq z$ for all $z \in$ $\operatorname{Fix}\left(G_{i}\right) \backslash \operatorname{Fix}\left(G_{i+1}\right)$, a contradiction. This implies $x=\sum_{\sigma \in G_{i}} m_{\sigma} x_{\sigma}$ as required.

Corollary 9. Let $A$ be a central division algebra over $F$ containing a maximal subfield $M$ with non-trivial solvable $G=\operatorname{Aut}_{F}(M)$. Then:

(i) A contains the cyclic division algebra $\left(M / \operatorname{Fix}\left(\sigma_{1}\right), \sigma_{1}, c_{0}\right)$ of prime degree $q_{0}$ over $\operatorname{Fix}\left(\sigma_{1}\right)$ as a subalgebra.

(ii) There is a non-central element $t_{0} \in A$ such that $t_{0}^{q_{0}} \in \operatorname{Fix}\left(\sigma_{1}\right)^{\times}$and $t_{0}^{m} \notin \operatorname{Fix}\left(\sigma_{1}\right)$ for all $1 \leq m<q_{0}$.

Here, $q_{0}$ is the order of the cyclic subgroup $G_{1}$ of the normal subseries (9) of $G$.

Additionally, we obtain the following straightforward observations:

Corollary 10. Let $A_{i}$ be as in (11) of Theorem $7, i \in\{0, \ldots, k\}$.

(i) $A_{i}$ is a generalized cyclic algebra over $Z_{i}=\operatorname{Fix}\left(G_{i}\right)$ of degree

$$
\operatorname{deg}\left(A_{i-1}\right) q_{i-1}=\prod_{l=0}^{i-1} q_{l}
$$

and

$$
M=Z_{0} \supset \ldots \supset Z_{k-1} \supset Z_{k} \supset F .
$$

(ii) $M / Z_{i}$ is a Galois extension and $M$ is a maximal subfield of $A_{i}$. 
Proof. (i) and (ii): $A_{i}$ is a generalized cyclic algebra as defined in 1.2 , Since $G_{i-1} \leq$ $G_{i}$ we have

$$
Z_{i}=\operatorname{Fix}\left(G_{i}\right) \subset \operatorname{Fix}\left(G_{i-1}\right)=Z_{i-1},
$$

for all $i \in\{1, \ldots, k\}$. We know that $A_{i} \operatorname{has} \operatorname{deg}\left(A_{i}\right)=\operatorname{deg}\left(A_{i-1}\right) q_{i-1}$ over its center. By induction we obtain the assertion.

(ii) is trivial by Theorem 7 .

Hence even if a central division algebra $A$ over $F$ is a noncrossed product, if $A$ contains a maximal field extension $M$ with non-trivial solvable $G=\operatorname{Aut}_{F}(M)$ then it contains a chain of generalized cyclic division algebras:

Corollary 11. Let $M / F$ be a field extension of degree $n$ with non-trivial solvable $G=\operatorname{Aut}_{F}(M)$, and $A$ a central division algebra over $F$ with maximal subfield $M$. Then $A$ contains a chain of generalized cyclic division algebras $A_{i}$ over intermediate fields $Z_{i}=\operatorname{Fix}\left(G_{i}\right)$ of $M / F$ as in (11). Here, $q_{i}$ is the order of the cyclic factor group $G_{i+1} / G_{i}$ of the normal subseries (9) of $G$.

If $A$ is a division algebra in the above setup then $G$ solvable implies that $A^{\times}$ contains an solvable subgroup:

Lemma 12. Suppose $A$ is a central division algebra over $F$. If $A$ contains a maximal subfield $M / F$ with solvable $G=\operatorname{Aut}_{F}(M)$ then $A^{\times}$contains an solvable subgroup. If $M / F$ is Galois, i.e. A a $G$-crossed product algebra, then this solvable subgroup is irreducible.

Proof. As noted in [6, Lemma 1] (where $M / F$ is Galois, but the argument is the same), $N=\bigcup_{\sigma \in G} M^{\times} x_{\sigma} \subset A^{\times}$and it is easy to see that $M^{\times}$is a normal subgroup of $N$, and that $N$ is the normalizer of $M^{\times}$in $A^{\times}$. Therefore $N / M^{\times} \cong G$ as in Lemma 1 (iii) and if $G$ is solvable as assumed in later sections, we see that in fact $N$ is a solvable subgroup of $A^{\times}$. Since $N=\bigcup M^{\times} x_{\sigma}$, if $M / F$ is Galois, i.e. $A$ a $G$-crossed product algebra, then $N$ is irreducible, i.e. the $F$-algebra generated by elements of $N, F[N]$, is $A$ by [6, Lemma 1].

Our next result generalizes [12, (9)] and characterizes all the algebras with a maximal subfield $M / F$ that have a solvable automorphism group $G=\operatorname{Aut}_{F}(M)$ via generalized cyclic algebras:

Theorem 13. Let $M / F$ be a field extension of degree $n$ with non-trivial $G=$ $\operatorname{Aut}_{F}(M)$, and $A$ be a central simple algebra over $F$ with maximal subfield $M$. Then $G$ is solvable if there exists a chain of subalgebras

$$
M=A_{0} \subset A_{1} \subset \ldots \subset A_{k} \subset A
$$


of $A$ which all have maximal subfield $M$, where $A_{k}$ is a $G$-crossed product algebra over $\operatorname{Fix}(G)$, and where

$$
A_{i+1} \cong A_{i}\left[t_{i} ; \tau_{i}\right] / A_{i}\left[t_{i} ; \tau_{i}\right]\left(t_{i}^{q_{i}}-c_{i}\right),
$$

for all $i \in\{0, \ldots, k-1\}$, with

(i) $q_{i}$ a prime,

(ii) $\tau_{i}$ an $F$-automorphism of $A_{i}$ of inner order $q_{i}$ which restricts to an automorphism $\sigma_{i+1} \in G$, and

(iii) $c_{i} \in \operatorname{Fix}\left(\tau_{i}\right)^{\times}$.

Proof. Suppose there exists a chain of algebras $A_{i}, i \in\{0, \ldots, k\}$ satisfying the above assumptions. Put $G_{k}=G$. Since each $A_{i}$ has center $Z_{i}=Z_{i-1} \cap \operatorname{Fix}\left(\tau_{i-1}\right)$, so that by induction

$$
Z_{i}=\operatorname{Fix}\left(\tau_{0}\right) \cap \operatorname{Fix}\left(\tau_{1}\right) \cap \cdots \cap \operatorname{Fix}\left(\tau_{i-1}\right) \supset F,
$$

$M / Z_{i}$ is a Galois extension contained in $A_{i}$. Put $G_{i}=\operatorname{Gal}\left(M / Z_{i}\right)$, then each $A_{i}$ is a $G_{i}$-crossed product algebra. In particular, $G_{i}$ is a subgroup of $G_{i+1}$.

We use induction to prove that each $G_{i}$, thus $G$, is a solvable group.

For $i=1$,

$$
A_{1} \cong M\left[t_{0} ; \sigma_{1}\right] / M\left[t_{0} ; \sigma_{1}\right]\left(t_{0}^{q_{0}}-c_{0}\right)
$$

is a cyclic algebra of degree $q_{0}$ over $\operatorname{Fix}\left(\sigma_{1}\right) . G_{1}=<\sigma_{1}>$ is a cyclic group of prime order $q_{0}$ and therefore solvable.

We assume as induction hypothesis that if there exists a chain

$$
M=A_{0} \subset \ldots \subset A_{j}
$$

of algebras such that (20) holds for all $i \in\{0, \ldots, j-1\}, j \geq 1$, then $G_{j}$ is solvable.

For the induction step we take a chain of algebras $M=A_{0} \subset \ldots \subset A_{j} \subset A_{j+1}$,

$$
A_{i+1} \cong A_{i}\left[t_{i} ; \tau_{i}\right] / A_{i}\left[t_{i} ; \tau_{i}\right]\left(t_{i}^{q_{i}}-c_{i}\right)
$$

where $\tau_{i}$ is an automorphism of $A_{i}$ of inner order $q_{i}$ which induces an automorphism $\sigma_{i+1} \in G, c_{i} \in \operatorname{Fix}\left(\tau_{i}\right)$ is invertible and $q_{i}$ is prime, for all $i \in\{0, \ldots, j\}$. By the induction hypothesis, $G_{j}$ is a solvable group.

We show that $G_{j+1}$ is solvable: $t_{j}$ is an invertible element of

$$
A_{j+1} \cong A_{j}\left[t_{j} ; \tau_{j}\right] / A_{j}\left[t_{j} ; \tau_{j}\right]\left(t_{j}^{q_{j}}-c_{j}\right)
$$

with inverse $c_{j}^{-1} t_{j}^{q_{j}-1}$.

$A_{j}$ is a $G_{j}$-crossed product algebra over $Z_{j}$ with maximal subfield $M$. The $F$ automorphism $\tau_{j}$ on $A_{j}$ satisfies $t_{j} l=\tau_{j}(l) t_{j}$ for all $l \in A_{j}$ which implies the inner automorphism

$$
I_{t_{j}}: A \rightarrow A, d \mapsto t_{j} d t_{j}^{-1}
$$

restricts to $\tau_{j}$ on $A_{j}$ and to $\sigma_{j+1}$ on $M$. 
For any $\sigma \in G$ there exists an invertible $x_{\sigma} \in A$ such that the inner automorphism

$$
I_{x_{\sigma}}: A \rightarrow A, y \mapsto x_{\sigma} y x_{\sigma}^{-1}
$$

restricted to $M$ is $\sigma$. Hence we have $x_{\sigma_{j+1}}=t_{j}$ with $x_{\sigma_{j+1}}$ as defined in Lemma 1. We know that $\left\{1, t_{j}, \ldots, t_{j} q_{j}-1\right\}$ is a basis for $A_{j+1}$ as a left $A_{j}$-module. By (5) we have $x_{\sigma_{j+1}^{2}}=a_{1} t_{j}{ }^{2}, x_{\sigma_{j+1}^{3}}=a_{2} t_{j}{ }^{3}, \ldots$ for suitable $a_{i} \in M^{\times}$, so that w.l.o.g. $\left\{1, x_{\sigma_{j+1}}, \ldots, x_{\sigma_{j+1}^{q_{j}-1}}\right\}$ is a basis for $A_{j+1}$ as a left $A_{j}$-module.

Since $A_{j}$ is a $G_{j}$-crossed product algebra, it has an $M$-basis $\left\{x_{\rho} \mid \rho \in G_{j}\right\}$, and hence $A_{j+1}$ has basis

$$
\left\{x_{\rho} x_{\sigma_{j+1}^{i}} \mid \rho \in G_{j}, 0 \leq i \leq q_{j}-1\right\}
$$

as $M$-vector space.

Additionally, $x_{\rho} x_{\sigma_{j+1}^{i}} \in M^{\times} x_{\rho \sigma_{j+1}^{i}}$ by Lemma 1 (iii) and thus $A_{j+1}$ has the $M$ basis

$$
\left\{x_{\rho \sigma_{j+1}^{i}} \mid \rho \in G_{j}, 0 \leq i \leq q_{j}-1\right\} .
$$

$A_{j+1}$ is a $G_{j+1}$-crossed product algebra and thus also has the $M$-basis $\left\{x_{\sigma} \mid \sigma \in\right.$ $\left.G_{j+1}\right\}$. We use these two basis to show that $G_{j+1}=G_{j}<\sigma_{j+1}>$ : Write

$$
x_{\rho \sigma_{j+1}^{i}}=\sum_{\sigma \in G_{j+1}} m_{\sigma} x_{\sigma}
$$

for some $m_{\sigma} \in M$, not all zero. Then

$$
x_{\rho \sigma_{j+1}^{i}} m=\sum_{\sigma \in G_{j+1}} m_{\sigma} x_{\sigma} m=\sum_{\sigma \in G_{j+1}} m_{\sigma} \sigma(m) x_{\sigma},
$$

and

$$
x_{\rho \sigma_{j+1}^{i}} m=\rho \sigma_{j+1}^{i}(m) x_{\rho \sigma_{j+1}^{i}}=\rho \sigma_{j+1}^{i}(m) \sum_{\sigma \in G_{j+1}} m_{\sigma} x_{\sigma},
$$

for all $m \in M$. Let $\sigma \in G_{j+1}$ be such that $m_{\sigma} \neq 0$, then in particular

$$
m_{\sigma} \sigma(m) x_{\sigma}=\rho \sigma_{j+1}^{i}(m) m_{\sigma} x_{\sigma},
$$

for all $m \in M$, that is $\sigma=\rho \sigma_{j+1}^{i}$. This means that $\left\{\rho \sigma_{j+1}^{i} \mid \rho \in G_{j}, 0 \leq i \leq\right.$ $\left.q_{j}-1\right\} \subset G_{j+1}$. Both sets have the same size so must be equal and we conclude $G_{j+1}=G_{j}<\sigma_{j+1}>$.

Finally we prove $G_{j}$ is a normal subgroup of $G_{j+1}$ : the inner automorphism $I_{x_{\sigma_{j+1}}}$ restricts to the $F$-automorphism $\tau_{j}$ of $A_{j}$. In particular, this implies

$$
x_{\sigma_{j+1}} x_{\rho} x_{\sigma_{j+1}}^{-1} \in A_{j},
$$

for all $\rho \in G_{j}$. Furthermore,

$$
x_{\sigma_{j+1} \rho \sigma_{j+1}^{-1}} \in M^{\times} x_{\sigma_{j+1}} x_{\rho} x_{\sigma_{j+1}^{-1}}=M^{\times} x_{\sigma_{j+1}} x_{\rho} x_{\sigma_{j+1}}^{-1} \subset A_{j},
$$

for all $\rho \in G_{j}$ by Lemma 1 . 
Hence $\sigma_{j+1} \rho \sigma_{j+1}^{-1} \in G_{j}$ because $A_{j}$ is a $G_{j}$-crossed product algebra. Similarly, we see $\sigma_{j+1}^{r} \rho \sigma_{j+1}^{-r} \in G_{j}$ for all $r \in \mathbb{N}$. Let $g \in G_{j+1}$ be arbitrary and write $g=h \sigma_{j+1}^{r}$ for some $h \in G_{j}, r \in\left\{0, \ldots, q_{j}-1\right\}$ which we can do because $G_{j+1}=G_{j}<\sigma_{j+1}>$. Then

$$
g \rho g^{-1}=\left(h \sigma_{j+1}^{r}\right) \rho\left(h \sigma_{j+1}^{r}\right)^{-1}=h\left(\sigma_{j+1}^{r} \rho \sigma_{j+1}^{-r}\right) h^{-1} \in G_{j},
$$

for all $\rho \in G_{j}$ so $G_{j}$ is indeed normal.

It is well known that a group $G$ is solvable if and only if given a normal subgroup $H$ of $G$, both $H$ and $G / H$ are solvable. It is clear now that $G_{j+1} / G_{j}$ is cyclic and hence solvable, which implies $G_{j+1}$ is solvable as required.

\section{Solvable Crossed PRoduct algebras}

We keep the assumptions from the previous section, but from now on we focus on the case that $M / F$ is a Galois extension, i.e. now $A$ is a $G$-crossed product algebra.

We obtain the next result as a special case of Theorem 7 .

Theorem 14. Let $M / F$ be a Galois field extension of degree $n$ with non-trivial solvable $G=\operatorname{Aut}_{F}(M)$, and $A$ a central simple algebra of degree $n$ over $F$ with maximal subfield $M$. Then $A$ is a $G$-crossed product algebra and there exists a chain of subalgebras

$$
M=A_{0} \subset A_{1} \subset \ldots \subset A_{k}=M(G)=A,
$$

of $A$ which are generalized cyclic algebras of degree $\prod_{l=0}^{i-1} q_{l}$ over $Z_{i}=\operatorname{Fix}\left(G_{i}\right)$ of the type

$$
A_{i+1} \cong A_{i}\left[t_{i} ; \tau_{i}\right] / A_{i}\left[t_{i} ; \tau_{i}\right]\left(t_{i}^{q_{i}}-c_{i}\right)
$$

for all $i \in\{0, \ldots, k-1\}$, satisfying (i), (ii), (iii) in Theorem 7 . Additionally the following holds for all $i \in\{1, \ldots, k\}$ :

(iv) $Z_{i-1} / Z_{i}$ has prime degree $q_{i-1}$.

Proof. $A_{k}=M(G)=A$ by Theorem 2 and thus $Z_{k}=F$. It remains to prove (iv): $Z_{i-1} / Z_{i}$ is a proper field extension for all $i \in\{1, \ldots, k\}$ by the Fundamental Theorem of Galois Theory, because $G_{i-1}$ is a proper subgroup of $G_{i}$. We have $n=|G|=\prod_{l=0}^{k-1} q_{l}$ is the decomposition of $n$ as a product of $k$ primes by (18). Also

$$
n=[M: F]=\left[M: Z_{1}\right] \cdots\left[Z_{k-1}: F\right] \text {, }
$$

and so $\left[Z_{i-1}: Z_{i}\right]=q_{\pi(i-1)}$ for all $i \in\{1, \ldots, k\}$ where $\pi$ is a permutation of $\{0, \ldots, k-1\}$. Hence $\left[Z_{i-1}: Z_{i}\right]$ is prime for all $i \in\{1, \ldots, k\}$. We now prove $q_{i-1}=q_{\pi(i-1)}$ for all $i \in\{1, \ldots, k\}$ : Suppose towards a contradiction $j \in\{1, \ldots, k\}$ is such that $\left[Z_{j-1}: Z_{j}\right]=q_{\pi(j-1)} \neq q_{j-1}$, where we take $j$ to be as small as possible. 
The dimension of $A_{j}$ over $Z_{j}$ is

$$
\prod_{l=0}^{j-1} q_{l}^{2}=\left[M: Z_{j}\right]\left|G_{j}\right|=\left[M: Z_{j}\right]\left(\prod_{l=0}^{j-1} q_{l}\right) .
$$

Since

$$
\left[M: Z_{j}\right]=\prod_{l=0}^{j-1}\left[Z_{l}: Z_{l+1}\right]
$$

we obtain

$$
\left[M: Z_{j}\right]\left(\prod_{l=0}^{j-1} q_{l}\right)=\left(\prod_{l=0}^{j-1} q_{\pi(l)}\right)\left(\prod_{l=0}^{j-1} q_{l}\right)= \begin{cases}\left(\prod_{l=0}^{j-2} q_{l}\right)^{2} q_{\pi(j-1)} q_{j-1} & \text { if } j \geq 2, \\ q_{\pi(0)} q_{0} & \text { if } j=1\end{cases}
$$

where we have used the minimality of $j$. Since the $q_{l}$ are prime and $q_{j-1} \neq q_{\pi(j-1)}$, this implies that the dimension of $A_{j}$ over $Z_{j}$ is not a square, a contradiction. Thus $\left[Z_{i-1}: Z_{i}\right]=q_{i-1}$ for all $i \in\{1, \ldots, k\}$.

In general, it is not always easy to decide if a given crossed product algebra is a division algebra or not.

Theorem 15. In the setup of Theorem 14, the solvable crossed product algebra $A$ is a division algebra if and only if

$$
b \tau_{i}(b) \cdots \tau_{i}^{q_{i}-1}(b) \neq c_{i}
$$

for all $b \in A_{i}$ and $i \in\{0, \ldots, k-1\}$.

Proof. If $A$ is a division algebra then so are all the subalgebras $A_{i}, i \in\{0, \ldots, k-1\}$. In particular, this means that $t_{i}^{q_{i}}-c_{i} \in A_{i}\left[t_{i} ; \tau_{i}\right]$ is an irreducible twisted polynomial for all $i \in\{0, \ldots, k-1\}$, i.e.

$$
b \tau_{i}(b) \cdots \tau_{i}^{q_{i}-1}(b) \neq c_{i}
$$

for all $b \in A_{i}[$ [, 1.3.16].

Conversely suppose (22) holds for all $b \in A_{i}$ and $i \in\{0, \ldots, k-1\}$. We prove by induction that then $A_{i}$ is a division algebra for all $i \in\{0, \ldots, k\}$, thus in particular so is $A=A_{k}: A_{0}=M$ is a field. Assume as induction hypothesis that $A_{j}$ is a division algebra for some $j \in\{0, \ldots, k-1\}$. By the proof of Theorem $7, \tau_{j}^{q_{j}}$ is the inner automorphism $I_{c_{j}}(z)=c_{j} z c_{j}^{-1}$ on $A_{j}$. Therefore

$$
A_{j+1} \cong A_{j}\left[t_{j} ; \tau_{j}\right] / A_{j}\left[t_{j} ; \tau_{j}\right]\left(t_{j}^{q_{j}}-c_{j}\right)
$$

is a division algebra since $t_{j}^{q_{j}}-c_{j} \in A_{j}\left[t_{j} ; \tau_{j}\right]$ is irreducible by [7, 1.3.16], because by assumption

$$
b \tau_{j}(b) \cdots \tau_{j}^{q_{j}-1}(b) \neq c_{j}
$$

for all $b \in A_{j}$. Thus $A_{i}$ is a division algebra for all $i \in\{0, \ldots, k\}$ by induction. 
The next result follows from Theorem 13. It generalizes [12, (9)] and characterizes solvable crossed product algebras via generalized cyclic algebras:

Corollary 16. Let $A$ be a crossed product algebra of degree $n$ over $F$ with maximal subfield $M$ such that $M / F$ is a Galois field extension. Then $G=\operatorname{Gal}(M / F)$ is solvable if there exists a chain of subalgebras

$$
M=A_{0} \subset A_{1} \subset \ldots \subset A_{k}=A
$$

of $A$ which all have maximal subfield $M$, and are generalized cyclic algebras

$$
A_{i+1} \cong A_{i}\left[t_{i} ; \tau_{i}\right] / A_{i}\left[t_{i} ; \tau_{i}\right]\left(t_{i}^{q_{i}}-c_{i}\right),
$$

over their centers for all $i \in\{0, \ldots, k-1\}$, where $q_{i}$ is a prime, $\tau_{i}$ is an $F$ automorphism of $A_{i}$ of inner order $q_{i}$ which restricts to an automorphism $\sigma_{i+1} \in G$, and $c_{i} \in \operatorname{Fix}\left(\tau_{i}\right)^{\times}$.

Remark 17. Let $M / F$ be a finite Galois field extension with non-trivial solvable Galois group $G$ and $A$ a solvable crossed product algebra over $F$ with maximal subfield $M$.

A close inspection of Albert's proof [1, p. 182-187] shows that he constructs the same chain of algebras

$$
A_{i+1} \cong A_{i}\left[t_{i} ; \tau_{i}\right] / A_{i}\left[t_{i} ; \tau_{i}\right]\left(t_{i}^{q_{i}}-c_{i}\right)
$$

inside a solvable crossed product $A$ as we obtain in Theorem 14, but they are not explicitly identified as generalized cyclic algebras. We also obtain a converse of Albert's statement (Corollary 16).

Theorem 14 also tells us something about the existence of $n$-central elements in a solvable crossed product algebra $A$, as $t_{k-1}$ is a $q_{k-1}$-central element in $A$. Recall that for a central simple algebra $A$ over $F$ whose degree is a multiple of $n, u \in A \backslash F$ is called an $n$-central element if $u^{n} \in F^{\times}$and $u^{m} \notin F$ for all $1 \leq m<n$. The $n$-central elements play an important role in the structure of central simple algebras.

Corollary 18. Let $A$ be a solvable $G$-crossed product division algebra over $F$. Then

$$
A \cong D[t ; \tau] / D[t ; \tau]\left(t^{q}-c\right)=(D, \tau, c)
$$

is a generalized cyclic algebra, where $D$ is either a central simple algebra over its center and $\tau$ a suitable automorphism of $D$ of finite inner order which is a prime $q$, or $D$ is a cyclic Galois field extension of $F$ of prime degree $q$ with Galois group $G=<\tau>$.

A contains a q-central element.

Proof. The first assertion follows directly from Theorem 14. In particular, then $t \in A$ is a non-central element such that $t^{q} \in F^{\times}$and $t^{m} \notin F$ for all $1 \leq m<q$. 


\section{Some SIMPle CONSEQUENCES FOR ADMISSIBLE GROUPS}

A finite group $G$ is called admissible over a field $F$, if there exists a $G$-crossed product division algebra over $F[14]$.

Suppose $G$ is a finite solvable group, so that we have a chain of normal subgroups

$$
\{1\}=G_{0} \leq \ldots \leq G_{k}=G,
$$

where $G_{j} \triangleleft G_{j+1}$ and $G_{j+1} / G_{j}$ is cyclic of prime order $q_{j}$ for all $j \in\{0, \ldots, k-1\}$ as in (9) and (10).

Suppose $G$ is admissible over $F$. Then Theorem 14 shows that the subgroups $G_{i}$ of $G$ appearing in the chain of normal subgroups of $G$ are admissible over suitable intermediate fields of $M / F$ :

Theorem 19. Suppose $G$ is admissible over a field $F$. Then each $G_{i}$ in the above chain is admissible over the intermediate field $Z_{i}=\operatorname{Fix}\left(G_{i}\right)$ of $M / F$ and

$$
\left[Z_{i}: F\right]=\prod_{j=i}^{k-1} q_{j},
$$

$i \in\{1, \ldots, k\}$. In particular, $G_{k-1}$ is admissible over $Z_{k-1}=\operatorname{Fix}\left(G_{k-1}\right)$ which has prime degree $q_{k-1}$ over $F$.

Proof. As $G$ is $F$-admissible there exists a $G$-crossed product division algebra $A$ over $F$ and a chain of generalized cyclic division algebras

$$
M=A_{0} \subset \ldots \subset A_{k}=A
$$

over $F$, such that

$$
A_{i+1} \cong A_{i}\left[t_{i} ; \tau_{i}\right] / A_{i}\left[t_{i} ; \tau_{i}\right]\left(t_{i}^{q_{i}}-c_{i}\right)
$$

for all $i \in\{0, \ldots, k-1\}$, where $\tau_{i}$ is an automorphism of $A_{i}$ of inner order $q_{i}$ which restricts to an automorphism $\sigma_{i+1} \in G$ and $c_{i} \in \operatorname{Fix}\left(\tau_{i}\right)$ is invertible (Theorem 14). $A_{i}$ is a $G_{i}$-crossed product division algebra over $Z_{i}$ with maximal subfield $M$ and $M / Z_{i}$ is a Galois field extension with $\operatorname{Gal}\left(M / Z_{i}\right)=G_{i}$, i.e. $G_{i}$ is $Z_{i}$-admissible.

Example 20. Let $G=\mathbf{S}_{\mathbf{4}}$, then $G$ is $\mathbb{Q}$-admissible [14, Theorem 7.1], so there exists a central simple division algebra $D$ over $\mathbb{Q}$ with maximal subfield $M$, such that $M / F$ is a Galois field extension and $\operatorname{Gal}(M / F)=G$ is a finite solvable group. Let

$$
\{\operatorname{id}\} \triangleleft<(12)(34)>\triangleleft \mathbf{K} \triangleleft \mathbf{A}_{\mathbf{4}} \triangleleft \mathbf{S}_{4}
$$

be its subnormal series, where $\mathbf{K}$ is the Klein four-group and $\mathbf{A}_{\mathbf{4}}$ is the alternating group, and

$\mathbf{S}_{\mathbf{4}} / \mathbf{A}_{\mathbf{4}} \cong \mathbb{Z} / 2 \mathbb{Z}, \mathbf{A}_{\mathbf{4}} / \mathbf{K} \cong \mathbb{Z} / 3 \mathbb{Z}, \mathbf{K} /<(12)(34)>\cong \mathbb{Z} / 2 \mathbb{Z},<(12)(34)>/\{$ id $\} \cong \mathbb{Z} / 2 \mathbb{Z}$ 
By Corollary 16, there exists a corresponding chain of division algebras

$$
M=A_{0} \subset A_{1} \subset A_{2} \subset A_{3} \subset A_{4}=D
$$

over $\mathbb{Q}$, such that

$$
A_{i+1} \cong A_{i}\left[t_{i} ; \tau_{i}\right] / A_{i}\left[t_{i} ; \tau_{i}\right]\left(t_{i}^{q_{i}}-c_{i}\right)
$$

is a generalized cyclic division algebra over its center for all $i \in\{0,1,2,3\}$, where $\tau_{i}$ is an automorphism of $A_{i}$, whose restriction to $M$ is $\sigma_{i+1} \in G, c_{i} \in \operatorname{Fix}\left(\tau_{i}\right)$, and $\tau_{i}$ has inner order $2,2,3,2$ for $i=0,1,2,3$ respectively. Moreover, we have $q_{0}=q_{1}=q_{3}=2$ and $q_{2}=3$, and $A_{i}$ has degree $\prod_{l=0}^{i-1} q_{l}$ over its center $Z_{i}$ for all $i \in\{1,2,3,4\}$ by Theorem 14. In addition, by Theorem 19] we conclude:

(i) $\mathbf{A}_{\mathbf{4}}$ is admissible over the quadratic field extension $Z_{3}=\operatorname{Fix}\left(\mathbf{A}_{\mathbf{4}}\right) \subset M$ of $\mathbb{Q}$.

(ii) $\mathbf{K}$ is admissible over the field extension $Z_{2}=\operatorname{Fix}(\mathbf{K}) \subset M$ of $\mathbb{Q}$ of degree 6 .

(iii) $\left\langle(12)(34)>\right.$ is admissible over the field extension $Z_{1}=\operatorname{Fix}(<(12)(34)>$ )$\subset M$ of $\mathbb{Q}$ of degree 12 .

Schacher proved that for every finite group $G$, there exists an algebraic number field $F$ such that $G$ is admissible over $F$ [14, Theorem 9.1]. Combining this with Theorem 14 we obtain:

Corollary 21. Let $G$ be a finite solvable group. Then there exists an algebraic number field $F$ and a G-crossed product division algebra $A$ over $F$. Furthermore, there exists a chain of division algebras

$$
M=A_{0} \subset A_{1} \subset \ldots \subset A_{k}=A
$$

over F, such that

$$
A_{i+1} \cong A_{i}\left[t_{i} ; \tau_{i}\right] / A_{i}\left[t_{i} ; \tau_{i}\right]\left(t_{i}^{q_{i}}-c_{i}\right)
$$

is a generalized cyclic algebra over its center $Z_{i}$ for all $i \in\{0, \ldots, k-1\}$, satisfying the properties listed in Theorems 7 and 14.

In particular, each $G_{i}$ is admissible over $Z_{i}$.

Proof. Such a field $F$ and division algebra $D$ exist by [14, Theorem 9.1]. The assertion follows by Corollary [16.

In [16, Theorem 1], Sonn proved that a finite solvable group is admissible over $\mathbb{Q}$ if and only if all its Sylow subgroups are metacyclic, i.e. if every Sylow subgroup $H$ of $G$ has a cyclic normal subgroup $N$, such that $H / N$ is also cyclic. Combining this with Theorem 14 we conclude:

Corollary 22. Let $G$ be a finite solvable group such that all its Sylow subgroups are metacyclic. Then there exists a $G$-crossed product division algebra $A$ over $\mathbb{Q}$, and a chain of division algebras

$$
M=A_{0} \subset A_{1} \subset \ldots \subset A_{k}=A
$$


over $\mathbb{Q}$, such that

$$
A_{i+1} \cong A_{i}\left[t_{i} ; \tau_{i}\right] / A_{i}\left[t_{i} ; \tau_{i}\right]\left(t_{i}^{q_{i}}-c_{i}\right)
$$

is a generalized cyclic algebra over its center $Z_{i}$ for all $i \in\{0, \ldots, k-1\}$ satisfying the properties listed in Theorems 1 and 14.

In particular, each $G_{i}$ is admissible over the field extension $Z_{i}$ of $\mathbb{Q}$.

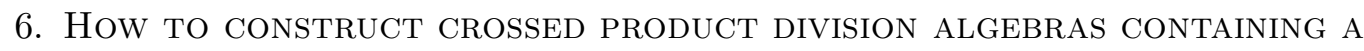 Given abelian Galois field extension as a maXimal SUbField}

For $S$ a unital ring and $\tau$ an injective endomorphism of $S$,

$$
f(t)=t^{q}-c \in S[t ; \tau]
$$

is an invariant twisted polynomial, if

$$
\tau^{q}(z) c=c \tau^{i}(z) \text { and } \tau(c)=c
$$

for all $z \in S, 0 \leq i<q$.

Let $M / F$ be a Galois field extension of degree $n$ with abelian Galois group $G=$ $\operatorname{Gal}(M / F)$. We now show how to canonically construct crossed product division algebras of degree $n$ over $F$ containing $M$ as a subfield. This generalizes a result by Albert in which $n=4$ and $G \cong \mathbb{Z}_{2} \times \mathbb{Z}_{2}$ [1, p. 186], cf. also [7, Theorem 2.9.55]: For $n=4$ every central division algebra containing a quartic abelian extension $M$ with Galois group $\mathbb{Z}_{2} \times \mathbb{Z}_{2}$ can be obtained this way [1, p. 186], that means as a generalized cyclic algebra $(D, \tau, c)$ with $D$ a quaternion algebra over its center.

Another way to construct such a crossed product algebra is via generic algebras, using a process going back to Amitsur and Saltman [3], described also in [7, 4.6].

As $G$ is a finite abelian group, we have a chain of subgroups

$$
\{1\}=G_{0} \leq \ldots \leq G_{k}=G,
$$

such that $G_{j} \triangleleft G_{j+1}$ and $G_{j+1} / G_{j}$ is cyclic of prime order $q_{j}>1$ for all $j \in\{0, \ldots, k-$ $1\}$. We use this chain to construct the algebras we want:

$G_{1}=<\sigma_{1}>$ is cyclic of prime order $q_{0}>1$ for some $\sigma_{1} \in G$. Let $\tau_{0}=\sigma_{1}$. Choose any $c_{0} \in F^{\times}$that satisfies

$$
z \tau_{0}(z) \cdots \tau_{0}^{q_{0}-1}(z) \neq c_{0}
$$

for all $z \in M$ and define

$$
f\left(t_{0}\right)=t_{0}^{q_{0}}-c_{0} \in M\left[t_{0} ; \tau_{0}\right] .
$$

Since $\tau_{0}$ has order $q_{0}$, we have $\tau_{0}^{q_{0}}(z) c_{0}=z c_{0}=c_{0} z$ for all $z \in M$, so that $f\left(t_{0}\right)$ is an invariant twisted polynomial and hence

$$
A_{1}=M\left[t_{0} ; \tau_{0}\right] / M\left[t_{0} ; \tau_{0}\right]\left(t_{0}^{q_{0}}-c_{0}\right)
$$


is an associative algebra which is cyclic of degree $q_{0}$ over Fix $\left(\tau_{0}\right)$. Moreover, $f\left(t_{0}\right)$ is irreducible by [7, 2.6.20 (i)] and therefore $A_{1}$ is a division algebra.

Now $G_{2} / G_{1}$ is cyclic of prime order $q_{1}$, say

$$
G_{2} / G_{1}=\left\{\sigma_{2}^{i} G_{1} \mid i \in \mathbb{Z}\right\}
$$

for some $\sigma_{2} \in G_{2}$ where $\sigma_{2}^{q_{1}} \in G_{1}$. As $\sigma_{2}^{q_{1}} \in G_{1}$ we have $\sigma_{2}^{q_{1}}=\sigma_{1}^{\mu}$ for some $\mu \in\left\{0, \ldots, q_{0}-1\right\}$. Define $c_{1}=l_{1} t_{0}^{\mu}$ for some $l_{1} \in F^{\times}$and define the map

$$
\tau_{1}: A_{1} \rightarrow A_{1}, \sum_{i=0}^{q_{0}-1} m_{i} t_{0}^{i} \mapsto \sum_{i=0}^{q_{0}-1} \sigma_{2}\left(m_{i}\right) t_{0}^{i}
$$

which is an automorphism of $A_{1}$ by a straightforward calculation.

Denote the multiplication in $A_{1}$ by $\circ$. Then

$$
\tau_{1}\left(c_{1}\right)=\sigma_{2}\left(l_{1}\right) t_{0}^{\mu}=l_{1} t_{0}^{\mu}=c_{1} .
$$

We have

$$
\tau_{1}^{q_{1}}\left(\sum_{i=0}^{q_{0}-1} m_{i} t_{0}^{i}\right) \circ c_{1}=\sum_{i=0}^{q_{0}-1} \sigma_{2}^{q_{1}}\left(m_{i}\right) t_{0}^{i} \circ l_{1} t_{0}^{\mu}=\sum_{i=0}^{q_{0}-1} l_{1} \sigma_{1}^{\mu}\left(m_{i}\right) t_{0}^{i} \circ t_{0}^{\mu}
$$

and

$$
c_{1} \circ \sum_{i=0}^{q_{0}-1} m_{i} t_{0}^{i}=l_{1} t_{0}^{\mu} \circ \sum_{i=0}^{q_{0}-1} m_{i} t_{0}^{i}=\sum_{i=0}^{q_{0}-1} l_{1} \sigma_{1}^{\mu}\left(m_{i}\right) t_{0}^{\mu} \circ t_{0}^{i}
$$

for all $m_{i} \in M$. Hence $\tau_{1}^{q_{1}}(z) \circ c_{1}=c_{1} \circ z$ for all $z \in A_{1}$ and $\tau_{1}\left(c_{1}\right)=c_{1}$, thus

$$
f\left(t_{1}\right)=t_{1}^{q_{1}}-c_{1} \in A_{1}\left[t_{1} ; \tau_{1}\right]
$$

is an invariant twisted polynomial and

$$
A_{2}=A_{1}\left[t_{1} ; \tau_{1}\right] / A_{1}\left[t_{1} ; \tau_{1}\right]\left(t_{1}^{q_{1}}-c_{1}\right)
$$

is a finite-dimensional associative algebra over $\operatorname{Fix}\left(\tau_{1}\right) \cap C\left(A_{1}\right)=\operatorname{Fix}\left(\tau_{1}\right) \cap \operatorname{Fix}\left(\tau_{0}\right) \supset$ $F[13$.

Again, $G_{3} / G_{2}$ is cyclic of prime order $q_{2}$, say

$$
G_{3} / G_{2}=\left\{\sigma_{3}^{i} G_{2} \mid i \in \mathbb{Z}\right\}
$$

for some $\sigma_{3} \in G$ with $\sigma_{3}^{q_{2}} \in G_{2}$. Write

$$
\sigma_{3}^{q_{2}}=\sigma_{2}^{\lambda_{1}} \sigma_{1}^{\lambda_{0}}
$$

for some $\lambda_{1} \in\left\{0, \ldots, q_{1}-1\right\}$ and $\lambda_{0} \in\left\{0, \ldots, q_{0}-1\right\}$. The map

$$
H_{\sigma_{3}}: A_{1} \rightarrow A_{1}, \sum_{i=0}^{q_{0}-1} m_{i} t_{0}^{i} \mapsto \sum_{i=0}^{q_{0}-1} \sigma_{3}\left(m_{i}\right) t_{0}^{i},
$$


is an automorphism of $A_{1}$ by a straightforward calculation. Define

$$
\tau_{2}: A_{2} \rightarrow A_{2}, \quad \sum_{i=0}^{q_{1}-1} x_{i} t_{1}^{i} \mapsto \sum_{i=0}^{q_{1}-1} H_{\sigma_{3}}\left(x_{i}\right) t_{1}^{i}\left(x_{i} \in A_{1}\right) .
$$

Then a straightforward calculation using that $H_{\sigma_{3}}$ commutes with $\tau_{1}$ and $H_{\sigma_{3}}\left(c_{1}\right)=$ $c_{1}$ shows that $\tau_{2}$ is an automorphism of $A_{2}$. Define

$$
c_{2}=l_{2} t_{0}^{\lambda_{0}} t_{1}^{\lambda_{1}}
$$

for some $l_{2} \in F^{\times}$. Denote the multiplication in $A_{i}$ by $\circ_{A_{i}}$ and let $x_{i}=\sum_{j=0}^{q_{0}-1} y_{i j} t_{0}^{j} \in$ $A_{1}, y_{i j} \in M, i \in\left\{0, \ldots, q_{1}-1\right\}$. Then

$$
\tau_{2}\left(c_{2}\right)=\tau_{2}\left(l_{2} t_{0}^{\lambda_{0}} t_{1}^{\lambda_{1}}\right)=H_{\sigma_{3}}\left(l_{2} t_{0}^{\lambda_{0}}\right) t_{1}^{\lambda_{1}}=l_{2} t_{0}^{\lambda_{0}} t_{1}^{\lambda_{1}}=c_{2} .
$$

Furthermore we have

$$
\begin{aligned}
\tau_{2}^{q_{2}}\left(\sum_{i=0}^{q_{1}-1} x_{i} t_{1}^{i}\right) \circ_{A_{2}} c_{2} & =\sum_{i=0}^{q_{1}-1} H_{\sigma_{3}}^{q_{2}}\left(x_{i}\right) t_{1}^{i} \circ_{A_{2}} l_{2} t_{0}^{\lambda_{0}} t_{1}^{\lambda_{1}} \\
& =\sum_{i=0}^{q_{1}-1} \sum_{j=0}^{q_{0}-1} \sigma_{3}^{q_{2}}\left(y_{i j}\right) t_{0}^{j} t_{1}^{i} \circ_{A_{2}} l_{2} t_{0}^{\lambda_{0}} t_{1}^{\lambda_{1}} \\
& =\sum_{i=0}^{q_{1}-1} \sum_{j=0}^{q_{0}-1} \sigma_{2}^{\lambda_{1}}\left(\sigma_{1}^{\lambda_{0}}\left(y_{i j}\right)\right) t_{0}^{j} t_{1}^{i} \circ_{A_{2}} l_{2} t_{0}^{\lambda_{0}} t_{1}^{\lambda_{1}} \\
& =\sum_{i=0}^{q_{1}-1}\left(\sum_{j=0}^{q_{0}-1} \sigma_{2}^{\lambda_{1}}\left(\sigma_{1}^{\lambda_{0}}\left(y_{i j}\right)\right) t_{0}^{j} \circ_{A_{1}} \tau_{1}^{i}\left(l_{2} t_{0}^{\lambda_{0}}\right)\right) t_{1}^{i} \circ_{A_{2}} t_{1}^{\lambda_{1}} \\
& =\sum_{i=0}^{q_{1}-1}\left(\sum_{j=0}^{q_{0}-1} l_{2} \sigma_{2}^{\lambda_{1}}\left(\sigma_{1}^{\lambda_{0}}\left(y_{i j}\right)\right) t_{0}^{j} \circ_{A_{1}} t_{0}^{\lambda_{0}}\right) t_{1}^{i} \circ_{A_{2}} t_{1}^{\lambda_{1}},
\end{aligned}
$$

and

$$
\begin{aligned}
c_{2} \circ A_{2} \sum_{i=0}^{q_{1}-1} x_{i} t_{1}^{i} & =l_{2} t_{0}^{\lambda_{0}} t_{1}^{\lambda_{1}} \circ_{A_{2}} \sum_{i=0}^{q_{1}-1} x_{i} t_{1}^{i}=\sum_{i=0}^{q_{1}-1}\left(l_{2} t_{0}^{\lambda_{0}} \circ_{A_{1}} \tau_{1}^{\lambda_{1}}\left(x_{i}\right)\right) t_{1}^{\lambda_{1}} \circ_{A_{2}} t_{1}^{i} \\
& =\sum_{i=0}^{q_{1}-1} \sum_{j=0}^{q_{0}-1}\left(l_{2} t_{0}^{\lambda_{0}} \circ_{A_{1}} \sigma_{2}^{\lambda_{1}}\left(y_{i j}\right) t_{0}^{j}\right) t_{1}^{\lambda_{1}} \circ_{A_{2}} t_{1}^{i} \\
& =\sum_{i=0}^{q_{1}-1}\left(\sum_{j=0}^{q_{0}-1} l_{2} \sigma_{1}^{\lambda_{0}}\left(\sigma_{2}^{\lambda_{1}}\left(y_{i j}\right)\right) t_{0}^{\lambda_{0}} \circ_{A_{1}} t_{0}^{j}\right) t_{1}^{\lambda_{1}} \circ_{A_{2}} t_{1}^{i} .
\end{aligned}
$$

Hence $\tau_{2}^{q_{2}}(z) \circ_{A_{2}} c_{2}=c_{2} \circ_{A_{2}} z$ for all $z \in A_{2}$ and $\tau_{2}\left(c_{2}\right)=c_{2}$, therefore

$$
f\left(t_{2}\right)=t_{2}^{q_{2}}-c_{2} \in A_{2}\left[t_{2} ; \tau_{2}\right]
$$

is an invariant twisted polynomial and thus

$$
A_{3}=A_{2}\left[t_{2} ; \tau_{2}\right] / A_{2}\left[t_{2} ; \tau_{2}\right]\left(t_{2}^{q_{2}}-c_{2}\right)
$$


is a finite-dimensional associative algebra over

$$
\operatorname{Fix}\left(\tau_{2}\right) \cap C\left(A_{2}\right)=\operatorname{Fix}\left(\tau_{0}\right) \cap \operatorname{Fix}\left(\tau_{1}\right) \cap \operatorname{Fix}\left(\tau_{2}\right) \supset F
$$

[13. Continuing in this manner we obtain a chain $M=A_{0} \subset \ldots \subset A_{k}$ of finitedimensional associative algebras

$$
A_{i+1}=A_{i}\left[t_{i} ; \tau_{i}\right] / A_{i}\left[t_{i} ; \tau_{i}\right]\left(t_{i}^{q_{i}}-c_{i}\right)
$$

over

$$
\operatorname{Fix}\left(\tau_{i}\right) \cap C\left(A_{i}\right)=\operatorname{Fix}\left(\tau_{0}\right) \cap \operatorname{Fix}\left(\tau_{1}\right) \cap \cdots \cap \operatorname{Fix}\left(\tau_{i}\right) \supset F,
$$

for all $i \in\{0, \ldots, k-1\}$, where $\tau_{0}=\sigma_{1}$ and $\tau_{i}$ restricts to $\sigma_{i+1}$ on $M$ for all $i \in\{0, \ldots, k-1\}$. Moreover,

$$
\left[A_{i}: M\right]=\left[A_{i}: A_{i-1}\right] \cdots\left[A_{1}: M\right]=\prod_{l=0}^{i-1} q_{l}
$$

hence

$$
\left[A_{k}: F\right]=\left(\prod_{l=0}^{k-1} q_{l}\right) n=n^{2},
$$

and $A_{k}$ contains $M$ as a subfield.

Lemma 23. For all $i \in\{0, \ldots, k-1\}, \tau_{i}: A_{i} \rightarrow A_{i}$ has inner order $q_{i}$.

Proof. The automorphism $\tau_{0}=\sigma_{1}: M \rightarrow M$ has inner order $q_{0}$.

Fix $i \in\{1, \ldots, k-1\}$. $A_{i}$ is finite-dimensional over $F$, so it is also finitedimensional over its center $C\left(A_{i}\right) \supset F$. Recall that $\tau_{i}^{q_{i}}(z) c_{i}=c_{i} z$ for all $z \in A_{i}$, in particular $\left.\tau_{i}^{q_{i}}\right|_{C\left(A_{i}\right)}=i d$. As $q_{i}$ is prime this means either $\left.\tau_{i}\right|_{C\left(A_{i}\right)}=i d$ or $\left.\tau_{i}\right|_{C\left(A_{i}\right)}$ has order $q_{i}>1$.

Assume that $\left.\tau_{i}\right|_{C\left(A_{i}\right)}=i d$, then $\tau_{i}$ is an inner automorphism of $A_{i}$ by the Theorem of Skolem-Noether, say $\tau_{i}(z)=u z u^{-1}$ for some invertible $u \in A_{i}$, for all $z \in A_{i}$. In particular $\tau_{i}(m)=\sigma_{i+1}(m)=u m u^{-1}$ for all $m \in M$. Write

$$
u=\sum_{j=0}^{q_{i-1}-1} u_{j} t_{i-1}^{j}
$$

for some $u_{j} \in A_{i-1}$, thus

$$
\begin{aligned}
\sigma_{i+1}(m) u & =\sigma_{i+1}(m) \sum_{j=0}^{q_{i-1}-1} u_{j} t_{i-1}^{j}=\sum_{j=0}^{q_{i-1}-1} u_{j} t_{i-1}^{j} m \\
& =\sum_{j=0}^{q_{i-1}-1} u_{j} \tau_{i-1}^{j}(m) t_{i-1}^{j}=\sum_{j=0}^{q_{i-1}-1} u_{j} \sigma_{i}^{j}(m) t_{i-1}^{j} .
\end{aligned}
$$

for all $m \in M$. Choose $\eta_{i}$ with $u_{\eta_{i}} \neq 0$ then

$$
\sigma_{i+1}(m) u_{\eta_{i}}=u_{\eta_{i}} \sigma_{i}^{\eta_{i}}(m),
$$


for all $m \in M$.

If $i=1$ we are done. If $i \geq 2$ then we can also write $u_{\eta_{i}}=\sum_{l=0}^{q_{i-2}-1} w_{l} t_{i-2}^{l}$ for some $w_{l} \in A_{i-2}$, therefore (24) yields

$$
\begin{aligned}
\sigma_{i+1}(m) \sum_{l=0}^{q_{i-2}-1} w_{l} t_{i-2}^{l} & =\sum_{l=0}^{q_{i-2}-1} w_{l} t_{i-2}^{l} \sigma_{i}^{\eta_{i}}(m)=\sum_{l=0}^{q_{i-2}-1} w_{l} \tau_{i-2}^{l}\left(\sigma_{i}^{\eta_{i}}(m)\right) t_{i-2}^{l} \\
& =\sum_{l=0}^{q_{i-2}-1} w_{l} \sigma_{i-1}^{l}\left(\sigma_{i}^{\eta_{i}}(m)\right) t_{i-2}^{l},
\end{aligned}
$$

for all $m \in M$. Choose $\eta_{i-1}$ with $w_{\eta_{i-1}} \neq 0$, then

$$
\sigma_{i+1}(m) w_{\eta_{i-1}}=w_{\eta_{i-1}} \sigma_{i-1}^{\eta_{i-1}}\left(\sigma_{i}^{\eta_{i}}(m)\right)
$$

for all $m \in M$.

Continuing in this manner we see that there exists $s \in M^{\times}$such that

$$
\sigma_{i+1}(m) s=s \sigma_{1}^{\eta_{1}}\left(\sigma _ { 2 } ^ { \eta _ { 2 } } \left(\cdots\left(\sigma_{i}^{\eta_{i}}(m) \cdots\right),\right.\right.
$$

for all $m \in M$, hence

$$
\sigma_{i+1}(m)=\sigma_{1}^{\eta_{1}}\left(\sigma _ { 2 } ^ { \eta _ { 2 } } \left(\cdots\left(\sigma_{i}^{\eta_{i}}(m) \cdots\right),\right.\right.
$$

for all $m \in M$ where $\eta_{j} \in\left\{0, \ldots, q_{j-1}-1\right\}$ for all $j \in\{1, \ldots, i\}$. But $\sigma_{i+1} \notin G_{i}$ and thus

$$
\sigma_{i+1} \neq \sigma_{1}^{\eta_{1}} \circ \sigma_{2}^{\eta_{2}} \circ \cdots \circ \sigma_{i}^{\eta_{i}}
$$

a contradiction.

It follows that $\left.\tau_{i}\right|_{C\left(A_{i}\right)}$ has order $q_{i}>1$. By the Skolem-Noether Theorem the kernel of the restriction map $\operatorname{Aut}\left(A_{i}\right) \rightarrow \operatorname{Aut}\left(C\left(A_{i}\right)\right)$ is the group of inner automorphisms of $A_{i}$, and so $\tau_{i}$ has inner order $q_{i}$.

Let us furthermore assume that each $c_{i}$ above, $i \in\{0, \ldots, k-1\}$, is successively chosen such that

$$
z \tau_{i}(z) \cdots \tau_{i}^{q_{i}-1}(z) \neq c_{i}
$$

for all $z \in A_{i}$, then using that $\tau_{i}$ has inner order $q_{i}$,

$$
f\left(t_{i}\right)=t_{i}^{q_{i}}-c_{i} \in A_{i}\left[t_{i} ; \tau_{i}\right]
$$

is an irreducible twisted polynomial by Lemma 23 and thus $A_{i+1}$ is a division algebra [7, 1.3.16].

Proposition 24. $C\left(A_{k}\right)=F$.

Proof. $F \subset C\left(A_{k}\right)$ by construction. Let now

$$
z=z_{0}+z_{1} t_{k-1}+\ldots+z_{q_{k-1}-1} t_{k-1}^{q_{k-1}-1} \in C\left(A_{k}\right)
$$


where $z_{i} \in A_{k-1}$. Then $z$ commutes with all $l \in A_{k-1}$, hence $l z_{i}=z_{i} \tau_{k-1}^{i}(l)$ for all $i \in$ $\left\{0, \ldots, q_{k-1}-1\right\}$. This implies $z_{0} \in C\left(A_{k-1}\right)$ and $z_{i}=0$ for all $i \in\left\{1, \ldots, q_{k-1}-1\right\}$, otherwise $z_{i}$ is invertible and $\tau_{k-1}^{i}$ is inner, a contradiction by Lemma 23. Thus $z=z_{0} \in C\left(A_{k-1}\right)$. A similar argument shows $z \in C\left(A_{k-1}\right)$ and continuing in this manner we conclude $z \in M=C\left(A_{0}\right)$.

Suppose for contradiction $z \notin F$, then $\rho(z) \neq z$ for some $\rho \in G$. Since the $\sigma_{i+1}$ were chosen so that they generate the cyclic factor groups $G_{i+1} / G_{i}$, we can write $\rho=\sigma_{1}^{i_{0}} \circ \sigma_{2}^{i_{1}} \circ \cdots \circ \sigma_{k}^{i_{k-1}}$ for some $i_{s} \in\left\{0, \ldots, q_{s}-1\right\}$. We have

$$
\begin{aligned}
t_{0}^{i_{0}} t_{1}^{i_{1}} \cdots t_{k-1}^{i_{k-1}} z & =\sigma_{1}^{i_{0}}\left(\sigma _ { 2 } ^ { i _ { 1 } } \left(\cdots\left(\sigma_{k}^{i_{k-1}}(z) \cdots\right) t_{0}^{i_{0}} t_{1}^{i_{1}} \cdots t_{k-1}^{i_{k-1}}\right.\right. \\
& =\rho(z) t_{0}^{i_{0}} t_{1}^{i_{1}} \cdots t_{k-1}^{i_{k-1}} \neq z t_{0}^{i_{0}} t_{1}^{i_{1}} \cdots t_{k-1}^{i_{k-1}},
\end{aligned}
$$

contradicting the assumption that $z \in C\left(A_{k}\right)$. Therefore $C\left(A_{k}\right) \subset F$.

This yields a recipe for constructing a $G$-crossed product division algebra $A=A_{k}$ over $F$ with maximal subfield $M$ provided it is possible to find suitable $c_{i}$ 's satisfying (25).

By Corollary 16, every abelian crossed product division algebra that is solvable can be obtained this way, starting with a suitable $M / F$.

\section{REFERENCES}

[1] A. A. Albert, Structure of algebras. Vol. 24, AMS 1939.

[2] A. S. Amitsur, Non-commutative cyclic fields. Duke Math. J. 21 (1954), 87105.

[3] A. S. Amitsur, D. J. Saltman Generic abelian crossed products and p-algebras. J. Alg. 51 (1978), $76-87$.

[4] C. Brown, PhD Thesis, University of Nottingham, in preparation.

[5] T. Hanke, A Direct Approach to Noncrossed Product Division Algebras. PhD Thesis, Universität Potsdam, 2011. Online at arXiv:1109.1580v1[math.RA]

[6] D. Kiani, M. Mahdavi-Hezavehi, Crossed product conditions for division algebras of prime power degree. J. Alg. 283 (2005), 222 - 231.

[7] N. Jacobson, "Finite-dimensional division algebras over fields." Springer Verlag, BerlinHeidelberg-New York, 1996.

[8] V. V. Kursov, V. I. Yanchevskii, Crossed products of simple algebras and their automorphism groups. Amer. Math. Soc. Transl. 154 (2) (1992), 75 - 80.

[9] T. Y. Lam, A first course in noncommutative rings. Springer-Verlag New York, 2nd ed., 2001.

[10] M. Motiee, A note on the existence of cyclic algebras in division algebras. Comm. Alg. 2016, DOI: 10.1080/00927872.2016.1264587

[11] J.-C. Petit, Sur certains quasi-corps généralisant un type d'anneau-quotient. Séminaire Dubriel. Algèbre et théorie des nombres 20 (1966 - 67), 1-18.

[12] J.-C. Petit, Sur les quasi-corps distributifes à base momogène. C. R. Acad. Sc. Paris 266 (1968), Série A, 402-404.

[13] S. Pumplün, Finite nonassociative algebras obtained from skew polynomials and possible applications to $(f, \sigma, \sigma)$-codes. To appear in Advances in Mathematics of Communications. Online at arXiv:1507.01491[cs.IT] 
[14] M. M. Schacher, Subfields of division rings, I. J. Algebra 9 (4) 1968, 451 - 477.

[15] R. D. Schafer, "An Introduction to Nonassociative Algebras." Dover Publ., Inc., New York, 1995.

[16] J. Sonn, $\mathbb{Q}$-admissibility of solvable groups. J. Algebra 84 (2), 1983, 411 - 419.

[17] O. Teichmüller, Über die sogenannte nichtkommutative Galoissche Theorie und die Relation $\xi \lambda, \mu, \nu \xi \lambda, \mu \nu, \pi \varepsilon, \lambda \mu, \nu, \pi=\pi \varepsilon, \lambda \mu, \nu, \pi$. (German) Deutsche Math. 5 (1940), 138 - 149.

[18] J.-P. Tignol, Generalized crossed products. Séminaire Mathématique (nouvelle série) 106 (1987), Université Catholique de Louvain, Louvain-la-Neuve, Belgium.

E-mail address: Christian.Brown@nottingham.ac.uk; susanne.pumpluen@nottingham.ac.uk

School of Mathematical Sciences, University of Nottingham, University Park, NotTingham NG7 2RD, United Kingdom 